\title{
Medindo os parâmetros de Stokes: Uma nova prática para ensino de óptica
}

\author{
Measuring the Stokes' parameters: A new practice for teaching optics
}

\author{
R. L. S. Lima ${ }^{1}$, E. S. Silva ${ }^{1}$, A. G. Rodrigues ${ }^{2}$, P. T. Araujo ${ }^{3}$, N. M. Barbosa Neto*1@ \\ ${ }^{1}$ Universidade Federal do Pará, Faculdade de Física, Instituto de Ciências Exatas e Naturais, Belém, PA, Brasil. \\ ${ }^{2}$ Universidade Federal do Pará, Instituto de Tecnologia, Belém, PA, Brasil. \\ ${ }^{3}$ University of Alabama, Department of Physics and Astronomy, Tuscaloosa, Alabama, United States
}

Recebido em 22 de abril de 2020. Revisado em 20 de julho de 2020. Aceito em 21 de julho de 2020.

\begin{abstract}
No presente trabalho mostramos a implementação de uma prática de laboratório utilizada para investigar os parâmetros de Stokes através da análise do comportamento da luz polarizada transmitida por elementos ópticos polarizantes. A prática foi implementada seguindo uma abordagem fundamentada em metodologia de aprendizagem baseada em projeto. Os diferentes estados de polarização da luz investigados foram obtidos através da construção de um simulador de polarização que é constituído de uma fonte de luz (laser em $670 \mathrm{~nm}$ ) combinado com diferentes elementos ópticos polarizantes. Mostramos que a técnica funciona de maneira bastante satisfatória para determinação dos parâmetros de Stokes e pode ser facilmente implementada como um projeto experimental ao longo do semestre em laboratórios avançados de ensino de óptica, suportando e expandindo os estudos teóricos do tema.
\end{abstract}

Palavras-chave: Parâmetros de Stokes, Polarização da luz, Experimentação.

\begin{abstract}
Here, we implement a physics teaching laboratory practice that aims to investigate the Stokes parameters through the analysis of polarized light transmitted by polarizing optical elements. The practice was implemented according to the project-based learning methodology. All the investigated states of polarized light were generated using a homemade polarization simulator, comprising a light source (laser emitting at $670 \mathrm{~nm}$ ) combined with different polarizing optical elements. We have shown that the practice is effective for obtaining the Stokes' parameters. Moreover, it can be easily designed as an advanced experimental project for optics teaching laboratories, with the purpose of confirming and advancing theoretical studies of the topic.
\end{abstract}

Keywords: Polarization of light, Stokes' parameters, Experimentation.

\section{Introdução}

Apesar de não pertencer ao grupo de disciplinas fundamentais da Física, a óptica figura como um dos ramos de grande importância desta ciência, uma vez que serve de suporte teórico e técnico para uma vasta quantidade de aplicações tecnológicas em nosso dia a dia, tais como lasers [1-3], sensores [1, 3, 4], fibras ópticas [3], processamento de imagens $[1,3,5]$, instrumentos de magnificação $[1,6]$, espectroscopia de materiais $[7,8]$ dentre outros. Em função disso, o ensino dessa disciplina em diferentes níveis do aprendizado é de fundamental importância, e o desenvolvimento de metodologias que sejam capazes de ir além da simples transmissão do conhecimento motivando os estudantes para área e fornecendo, além do conhecimento em óptica, um conjunto mais abrangente de habilidades e competências é algo bastante desejado.

A abordagem mais empregada em cursos básicos (ensino médio e primeiros anos de curso universitário), e que influencia a elaboração de boa parte do material didático

*Endereço de correspondência: newtonfisico@gmail.com disponível [9, 10], é baseada na análise dos fenômenos observados para a luz. Um aspecto positivo dessa abordagem é que em geral muitos tópicos são trabalhados a partir de experimentos simples, mas de grande potencial didático. Nestes o conhecimento é transmitido pela observação de fenômenos provenientes de experimentos demonstrativos [11-13].

No que se refere a disciplinas mais avançadas, estas se restringem a aulas teóricas onde o tratamento matemático rigoroso da eletrodinâmica de Maxwell é usado para explicar fenômenos tais como propagação, interferência, difração, espalhamento, absorção, dispersão e polarização da luz $[2,3,5]$. Neste sentido, a elaboração de experimentos que possam ser integrados ao nível de tratamento utilizado nos cursos avançados de óptica, via uma abordagem de aprendizado por projeto [14, 15], é uma necessidade. Uma das vantagens de elaborarmos tais experimentos é que estes possuem a característica de serem relativamente sofisticados tanto do ponto de vista técnico experimental quanto do ponto de vista teórico, o que contribui para o desenvolvimento de uma série de habilidades nos estudantes, tais como: domínio da meto- 
dologia científica, trabalho em equipe, planejamento, etc. Além disso, por serem elaborados visando sua aplicação em cursos avançados, eles se integram perfeitamente na metodologia baseada em projetos, exigindo dos estudantes um nível de comprometimento maior. Tal abordagem de aprendizado já foi testada antes em nível básico de um curso de eletricidade e magnetismo [16].

Efeitos relacionados à polarização são ricos em experimentos que exigem certo grau de sofisticação técnica e tratamento teórico. Neste trabalho propomos um experimento que pode ser montado em um curso de laboratório de óptica avançado (último ano do curso) com grande potencial didático, tanto para o ensino aprofundado do fenômeno da polarização quanto para o aprendizado de métodos experimentais. Este foi empregado em uma metodologia de aprendizado por projeto, aplicada a dois estudantes, com o objetivo de ensinar um tópico específico. O assunto abordado foi o formalismo de Stokes para luz polarizada, que foi escolhido por ser um dos principais formalismo utilizados para descrever o estado de polarização da luz e pelo fato de que nos últimos anos este vem sendo empregado em laboratórios de pesquisa para medir efeitos de anisotropia de polímeros emissores em solução [17], determinar a temperatura de transição de fase do tipo ordem-desordem em cristais líquidos [18,19], e determinar a componente circular de espalhamento Raman por moléculas [20]. O projeto consiste em duas fases: Na primeira, os estudantes realizam estudos sobre os fundamentos teóricos do formalismo de Stokes [21], via grupo de discussão e apresentação de seminários. Na segunda fase constroem e executam o experimento para demonstrar a validade do formalismo de Stokes. A técnica é construída usando tanto uma lâmina de quarto de onda comercial quanto com uma lâmina de onda caseira fabricada com fita adesiva como sugerido na referência [22]. Os resultados obtidos com as duas lâminas são comparados e discutidos.

\section{Fundamentos Teóricos}

\subsection{A elipse de polarização}

Das equações de Maxwell aplicadas ao espaço livre de cargas e correntes, obtemos equações de onda para os campos elétrico e magnético mutualmente interagentes, os quais oscilam ortogonalmente entre si no espaço e no tempo $[23,24]$. Ao tomarmos como referência para a polarização a dinâmica do campo elétrico, temos, nestas condições, como solução mais simples e geral para a equação de onda a forma de onda plana monocromática, i.e., um campo vetorial cujas componentes são perpendiculares a direção de propagação. Tomando o eixo z do sistema de referência do laboratório como a direção de propagação do feixe de luz, podemos escrever as componentes cartesianas do campo elétrico da onda plana como [21]:

$$
\begin{gathered}
\mathrm{E}_{\mathrm{x}}(\mathrm{z}, \mathrm{t})=\mathrm{E}_{0 \mathrm{x}} \cos (\tau) \\
\mathrm{E}_{\mathrm{y}}(\mathrm{z}, \mathrm{t})=\mathrm{E}_{0 \mathrm{y}} \cos (\tau+\delta)
\end{gathered}
$$

onde o termo $\tau=\omega \mathrm{t}-\mathrm{kz}$ é denominado de propagador, e descreve por completo as propriedades de propagação da onda tais como frequência de oscilação, número de onda, e direção de propagação, e $\delta$ é denominado de diferença de fase entre as componentes.

Apesar de ser a onda eletromagnética composta de dois campos vetoriais, para avaliarmos suas propriedades de polarização é suficiente observarmos um dos campos, já que estes se relacionam pelo produto vetorial $\vec{B}=\frac{1}{\mathrm{c}} \hat{k} \times \vec{E}$, sendo $\hat{k}$ o versor de propagação da onda [23]. Além disso, em geral os elementos de polarização (polarizadores, lâminas de onda, interfaces ar-vidro etc.) são meios dielétricos, que interagem de forma mais significativa com o campo elétrico da luz [1].

Similarmente ao movimento bidimensional de um projétil sob a ação da gravidade (onde uma equação de trajetória parabólica é obtida pela combinação dos movimentos nas coordenadas cartesianas a partir de sua mútua dependência com o tempo) é possível obtermos uma equação de elipse combinando as coordenadas cartesianas do campo elétrico através de sua dependência mútua com o propagador. Após algumas manipulações algébricas das equações (1) obtemos [9,21]:

$$
\frac{\mathrm{E}_{\mathrm{x}}^{2}(\mathrm{t})}{\mathrm{E}_{0 \mathrm{x}}^{2}}+\frac{\mathrm{E}_{\mathrm{y}}^{2}(\mathrm{t})}{\mathrm{E}_{0 \mathrm{y}}^{2}}-2 \frac{\mathrm{E}_{\mathrm{x}}(\mathrm{t}) \mathrm{E}_{\mathrm{y}}(\mathrm{t})}{\mathrm{E}_{0 \mathrm{x}} \mathrm{E}_{0 \mathrm{y}}} \cos \delta=\sin ^{2} \delta
$$

Esta elipse é denominada de elipse de polarização e representa a forma mais geral de polarização; uma elipse inclinada em relação aos eixos cartesianos [2,5,21]. Todos os casos especiais de polarização, tais como polarização linear $(\delta=0+\mathrm{n} \pi)$, circular $\left(\delta=\frac{\pi}{2}+\mathrm{n} \pi\right.$ e $\left.\mathrm{E}_{0 \mathrm{x}}=\mathrm{E}_{0 \mathrm{y}}\right)$ e elíptica $\left(\delta=\frac{\pi}{2}+\mathrm{n} \pi\right.$ e $\left.\mathrm{E}_{0 \mathrm{x}} \neq \mathrm{E}_{0 \mathrm{y}}\right)$, são obtidos a partir da elipse de polarização $[2,21]$.

\subsection{Formalismo de Stokes}

A descrição da polarização da luz através da elipse de polarização, equação (2), embora teoricamente simples, apresenta o inconveniente de ser experimentalmente impraticável. Sendo o período de oscilação da radiação eletromagnética visível da ordem de $10^{-15} \mathrm{~s}$ [2], este é muito rápido para ser detectado por dispositivos existentes em laboratório. Em outras palavras, não é possível medir diretamente o campo elétrico cuja dinâmica em um plano define a sua elipse de polarização. Isto ocorre, pois, os fotodetectores utilizam processos físicos que são correlacionados com a intensidade de campo incidente, sendo tais processos muito mais lentos do que o período da luz. Deste modo, é a intensidade, definida como uma média temporal do fluxo de energia transportada por uma onda eletromagnética [23], o observável medido.

Uma abordagem desenvolvida por Sir George Gabriel Stokes no século XIX para lidar com fenômenos relacionados às leis de interferência de Fresnel-Arago (1818) e aplicados a feixes parcialmente polarizados acabou por se mostrar extremamente útil para resolver o problema da medição da polarização [21,25]. Stokes criou um formalismo matemático, capaz de descrever os estados de 
polarização, que é baseado em quatro quantidades observáveis a partir de medidas de intensidade de luz. Ou seja, em medidas realizadas sobre o único observável da luz diretamente detectável em laboratório [3,21,26]. Para construir este formalismo toma-se a média temporal dos termos da elipse de polarização sobre um período de detecção, dito infinito se comparado ao período de oscilação da radiação. Esse procedimento nos permite expressar o comportamento médio da elipse de polarização como o conjunto de quatro parâmetros chamados parâmetros de Stokes. Estes parâmetros são escritos em termos das amplitudes das coordenadas cartesianas do campo elétrico e da diferença de fase entre elas [21,25,27]:

$$
\begin{gathered}
\mathrm{S}_{0}=\mathrm{E}_{0 \mathrm{x}}^{2}+\mathrm{E}_{0 \mathrm{y}}^{2} \propto \mathrm{I}_{\mathrm{x}}+\mathrm{I}_{\mathrm{y}} \\
\mathrm{S}_{1}=\mathrm{E}_{0 \mathrm{x}}^{2}-\mathrm{E}_{0 \mathrm{y}}^{2} \propto \mathrm{I}_{\mathrm{x}}-\mathrm{I}_{\mathrm{y}} \\
\mathrm{S}_{2}=2 \mathrm{E}_{0 \mathrm{x}} \mathrm{E}_{0 \mathrm{y}} \cos \delta \propto \mathrm{I}_{+45}-\mathrm{I}_{-45} \\
\mathrm{~S}_{3}=2 \mathrm{E}_{0 \mathrm{x}} \mathrm{E}_{0 \mathrm{y}} \sin \delta \propto \mathrm{I}_{\mathrm{D}}-\mathrm{I}_{\mathrm{E}}
\end{gathered}
$$

onde $I_{x}$ e $I_{y}$ são as intensidades de luz nos eixos cartesianos x-y do laboratório, $\mathrm{I}_{+45}$ e $\mathrm{I}_{-45}$ são as intensidades de luz sobre os eixos à $\pm 45^{\circ}$, em relação ao eixo $\mathrm{x}$, e $\mathrm{I}_{\mathrm{D}}$ e $\mathrm{I}_{\mathrm{E}}$ são intensidades de luz circularmente polarizada para direita (horário) e esquerda (anti-horário). Nas equações (3) o uso do sinal de proporcionalidade se deve ao fato de que os parâmetros de Stokes são escritos sem levar em conta as constantes existentes na definição de intensidade.

Construídos desta forma, os parâmetros de Stokes descrevem qualquer estado de polarização da luz. É fácil verificar o significado de cada um destes a partir das equações (3). Da equação (3a) verifica-se que o parâmetro $\mathrm{S}_{0}$, que é a soma das intensidades em eixos ortogonais, logo sempre positivo, associa-se a intensidade total da luz. Já o parâmetro $\mathrm{S}_{1}$, definido pela equação $(3 \mathrm{~b})$, é correlacionado com a diferença de intensidade entre as porções de luz polarizada na horizontal e vertical. Dessa maneira, se a componente na horizontal for maior, $0<\mathrm{S}_{1} \leq \mathrm{S}_{0}$, enquanto se a porção na vertical for maior, ocorre que $-\mathrm{S}_{0} \leq \mathrm{S}_{1}<0$, onde as igualdades são válidas para luz totalmente polarizada na horizontal e vertical, respectivamente. Já $S_{2}$ se relaciona com a diferença de intensidade entre as porções de luz polarizada sobre os eixos à $\pm 45^{\circ}$, respectivamente. Assim, se a componente à $+45^{\circ}$ for maior, $0<\mathrm{S}_{2} \leq \mathrm{S}_{0}$, e caso contrário, se a porção à $-45^{\circ}$ se sobressair, $-\mathrm{S}_{0} \leq \mathrm{S}_{2}<0$. Novamente, as igualdades asseguram os casos de polarizações totalmente projetadas sobre algum destes eixos. Da mesma maneira $\mathrm{S}_{3}$ associase com a diferença de intensidade das porções de luz polarizada circularmente para direita, cuja prevalência gera $0<\mathrm{S}_{3} \leq \mathrm{S}_{0}$, e para luz polarizada circularmente para esquerda, cuja prevalência gera $-\mathrm{S}_{0} \leq \mathrm{S}_{3}<0$. As igualdades também são válidas para os casos de polarizações totais sobre estas direções. Um caso especial é o da luz despolarizada, ou aleatoriamente polarizada, onde a intensidade da luz em qualquer uma dessas direções é a mesma, de modo que $\mathrm{S}_{1}=\mathrm{S}_{2}=\mathrm{S}_{3}=0$. É importante ressaltar que a convenção de polarização adotada aqui é definida em acordo a um observador que olha na direção de propagação da luz, como ilustrado na Figura 1.

Uma das vantagens imediatas do formalismo de Stokes sobre a elipse polarização [2,3,21] é que embora os estados de polarização definidos acima sejam estados de polarização para luz completamente polarizada, os parâmetros de Stokes são também capazes de descrever a luz não polarizada. Isto ocorre, pois, de modo geral, os parâmetros obedecem a desigualdade mostrada na equação (4) [21].

$$
\mathrm{S}_{0}^{2} \geq \mathrm{S}_{1}^{2}+\mathrm{S}_{2}^{2}+\mathrm{S}_{3}^{2}
$$

onde a igualdade é válida para o caso da luz totalmente polarizada. Note que $\mathrm{S}_{0}$ é relacionado a intensidade total da luz medida, cujo valor pode ser entendido como a soma das porções despolarizada e totalmente polarizada, esta última descrita por $\mathrm{S}_{1}^{2}+\mathrm{S}_{2}^{2}+\mathrm{S}_{3}^{2}$

Da desigualdade estabelecida na equação (4) define-se o grau de polarização da luz como:

$$
\mathrm{P}=\frac{\sqrt{\mathrm{S}_{1}^{2}+\mathrm{S}_{2}^{2}+\mathrm{S}_{3}^{2}}}{\mathrm{~S}_{0}}
$$

sendo $0 \leq \mathrm{P} \leq 1$. De acordo com a equação (5), $\mathrm{P}=$ 0 corresponde a luz completamente não polarizada enquanto $\mathrm{P}=1$, corresponde a luz totalmente polarizada. Valores intermediários de $\mathrm{P}$ correspondem a luz parcialmente polarizada.

\subsection{Matrizes de Mueller}

O formalismo das matrizes de Mueller é responsável por descrever a interação de luz polarizada com elementos ópticos polarizantes (polarizadores, lâminas de ondas, etc.) através da transformação linear do chamado vetor de

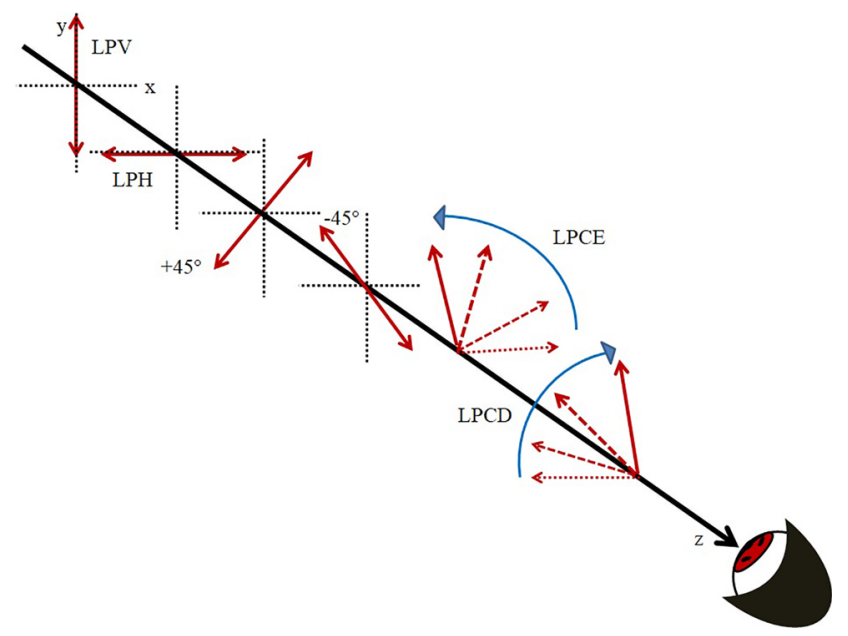

Figura 1: Esquema para ilustrar o observador que define a convenção adotada para os estados de polarização. Seguindo a direção z, eles são: LPV (luz linearmente polarizada na vertical); LPH (luz linearmente polarizada na horizontal); $\pm 45^{\circ}$ (luz linearmente polarizada a $\pm 45^{\circ}$ ); LCPD (luz circularmente polarizada para direita); LCPE (luz circularmente polarizada para esquerda) 
Stokes [3,5,21]. Em outras palavras, quando um feixe de luz polarizada atravessa um elemento óptico polarizante, os quatro parâmetros de Stokes que compõe o vetor de Stokes e que descrevem o estado de polarização de luz de saída são obtidos a partir de uma combinação linear dos parâmetros de Stokes da luz incidente, tendo como coeficientes termos associados ao elemento óptico [21]. Desta forma, o vetor de Stokes é modificado via uma operação matricial entre a matriz que representa a ação do elemento polarizante com o vetor de Stokes do feixe incidente:

$$
\underbrace{\left[\begin{array}{c}
\mathrm{S}_{0}^{\prime} \\
\mathrm{S}_{1}^{\prime} \\
\mathrm{S}_{2}^{\prime} \\
\mathrm{S}_{3}^{\prime}
\end{array}\right]}_{\mathrm{S}^{\prime}}=\underbrace{\left[\begin{array}{llll}
\mathrm{m}_{00} & \mathrm{~m}_{01} & \mathrm{~m}_{02} & \mathrm{~m}_{03} \\
\mathrm{~m}_{10} & \mathrm{~m}_{11} & \mathrm{~m}_{12} & \mathrm{~m}_{13} \\
\mathrm{~m}_{20} & \mathrm{~m}_{21} & \mathrm{~m}_{22} & \mathrm{~m}_{23} \\
\mathrm{~m}_{30} & \mathrm{~m}_{31} & \mathrm{~m}_{32} & \mathrm{~m}_{33}
\end{array}\right]}_{\mathrm{M}} \underbrace{\left[\begin{array}{c}
\mathrm{S}_{0} \\
\mathrm{~S}_{1} \\
\mathrm{~S}_{2} \\
\mathrm{~S}_{3}
\end{array}\right]}_{\mathrm{S}}
$$

A matriz $\mathrm{M}$ depende unicamente da forma como o elemento óptico interage com o feixe de luz, sendo cada elemento representado por sua própria matriz de Mueller. Para o problema abordado aqui duas matrizes de Mueller são usadas: 1) A matriz de Mueller para uma lâmina de quarto de onda (LQO), cujo eixo rápido é rotacionado, no sentido horário, de um ângulo $\theta$ em relação a um eixo de referência (coordenada y do referencial do laboratório, veja Figura 2) [3,21] e 2) A matriz de Mueller de um polarizador cujo eixo de transmissão se encontra na direção vertical (coordenada y do referencial do laboratório, veja Figura 2) [3,21].

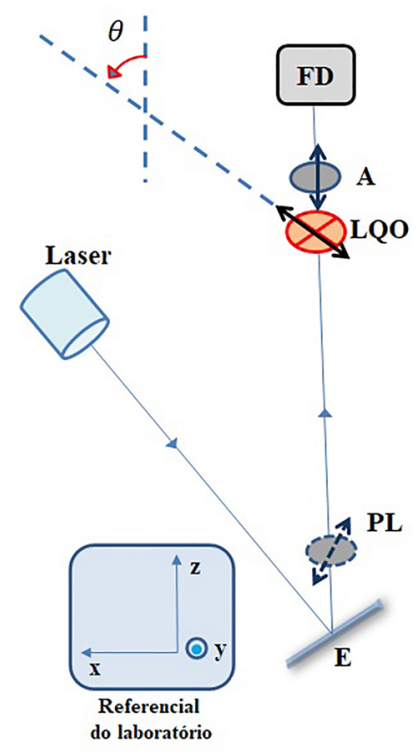

Figura 2: Esquema do aparato experimental utilizado. Seguindo a direção de propagação do feixe (linha sólida azul) tem-se: Laser; E: Espelho injetor; PL: Polarizadores lineares geradores dos estados de polarização; LQO: Lâmina de quarto de onda (comercial ou de fita adesiva); A: Analisador (polarizador linear); FD: fotodetector.
Combinando as matrizes de Muller para esses dois elementos ópticos, na ordem posicional mostrada na Figura 2 obtemos que a intensidade de luz medida para cada posição angular do eixo rápido da LQO é escrita em relação aos parâmetros de Stokes da luz incidente como $[21,25]$ :

$$
\begin{aligned}
& \mathrm{I}(\theta)=\frac{1}{2}[\underbrace{S_{0}}_{\text {intensidade total }}-\underbrace{\frac{\mathrm{S}_{1}(1+\cos 4 \theta)}{2}}_{\mathrm{LPV} \text { ou } \mathrm{LPH}} \\
& +\underbrace{\frac{\mathrm{S}_{2}}{2} \sin 4 \theta}_{ \pm 45^{\circ}}+\underbrace{\mathrm{S}_{3} \sin 2 \theta}_{\text {LPCE ou LPCD }}
\end{aligned}
$$

Em outras palavras, para um dado estado de polarização da luz incidente, o sistema composto pela LQO rotacionada mais o PLV fornece uma curva harmônica de intensidade, descrita pela equação (7). A curva, aqui batizada de curva de Stokes, pode ser interpretada como uma superposição de funções harmônicas ortogonais cujos coeficientes (ou amplitudes) dependem univocamente do estado de polarização inicial; i.e., uma superposição de estados de polarização fundamentais. Para o caso de uma curva de Stokes obtida para radiação monocromática, a maneira mais direta de obtermos os Parâmetros de Stokes é ajustando a curva experimental com a equação (7), pelo método de mínimos quadrados não linear, o que é facilmente realizado via softwares de análise de dados apropriados.

\section{Materiais e Métodos}

O sistema experimental empregado para adquirir o sinal de luz polarizado consiste em uma LQO, um PLV e um fotodetector. Como lâmina de quarto de onda foram empregadas uma lâmina de onda comercial acromática cujo intervalo de operação vai de $400 \mathrm{~nm}$ até $800 \mathrm{~nm}$, e uma lâmina de onda de fabricação caseira feita de fita adesiva seguindo os procedimentos da referência [22]. Para obtermos os diferentes estados de polarização da luz utilizada no estudo, construímos um simulador de luz polarizada que consiste um laser (emitindo em 670 $\mathrm{nm}$ ) polarizado linearmente na vertical e um conjunto de até dois polarizadores lineares. As polarizações lineares $\pm 45^{\circ}$ são obtidas transmitindo o feixe laser verticalmente polarizado através de um polarizador com o eixo de transmissão em $\pm 45^{\circ}$. Já a polarização linear horizontal é obtida transmitindo o feixe verticalmente polarizado primeiro por um polarizador a $45^{\circ}$ e depois por um segundo polarizador com eixo de transmissão na horizontal. O esquema do arranjo experimental utilizado é mostrado na Figura 2. Assim, é possível obter os seguintes estados de polarização básicos: Linear horizontal e vertical, linear $\pm 45^{\circ}$. Embora o laser utilizado nos experimentos possua polarização linear, também é possível realizar este experimento com lasers não polarizados, como por 
exemplo, lasers de He:Ne não polarizado ou apontadores laser. Todavia, nesta configuração polaroides lineares e/ou circulares devem ser utilizados para gerar o estado de polarização da luz. Além disso, é possível também obter luz polarizada refletindo luz não polarizada em uma superfície com configuração em ângulo de Brewster $[2,5,9,10]$.

Para detecção do sinal foi empregado um fotodetector de silício (SM1PDA thorlabs) conectado a um multímetro digital de fabricação caseira capaz de fazer média temporal do sinal. O multímetro utilizado foi feito com o uso de uma placa Arduino (modelo UNO) conectada a um computador [28]. Cada ponto na curva de Stokes e obtido a partir de uma média feita em 10 segundos de aquisição. No experimento o sinal luminoso é adquirido para diferentes posições angulares do eixo rápido da lâmina de quarto de onda que é rotacionada, em passos de $10^{\circ}$, em torno do eixo paralelo a direção de propagação do feixe de luz, direção z no sistema de referência da Figura 2.

Os ajustes teóricos das curvas experimentais, que fornecem os parâmetros de Stokes foram realizados com a equação (7), sendo estes realizados com o software ORIGIN 2018. Além disso, o grau de polarização para cada estado foi estimado segundo a equação (5), e seu erro, desvio padrão, advindos da sua dependência com os Parâmetros de Stokes relativos à $\mathrm{S}_{0}\left(\mathrm{~S}_{\mathrm{k}} / \mathrm{S}_{0}, \mathrm{k}=1,2,3\right)$, foram determinados segundo a regra de propagação de erros para funções de várias variáveis [29]. Vale ressaltar que tais desvios padrão são todos associados à natureza estatística do ajuste da curva teórica pelo método de mínimos quadrados não-linear, e não da dispersão sobre uma amostragem de vários experimentos. Como todos os valores aqui utilizados possuem a mesma natureza, a regra de propagação de erros permanece válida.

\section{Resultados e Discussão}

\subsection{Verificação experimental do formalismo de Stokes}

A seguir apresentamos os resultados experimentais juntamente com os ajustes teóricos realizados com a equação (7), obtidos para um feixe de luz linearmente polarizado na vertical e horizontal e linearmente polarizado a $\pm 45^{\circ}$. Para facilitar a comparação dos resultados obtidos, apresentamos os parâmetros de Stokes relativos à intensidade total. Em outras palavras as razões $\frac{S_{1}}{S_{0}}, \frac{S_{2}}{S_{0}}$ e $\frac{S_{3}}{S_{0}}$. Na Figura 3, mostramos a curva de Stokes obtida para luz linearmente polarizada na vertical (LPV) e horizontal (LPH), com o aparato experimental sendo montando com a lâmina de onda comercial

É observado que a curva de Stokes para a LPH, possui intensidade nula em cada múltiplo de $90^{\circ}$ para posição angular da LQO, o que está em acordo com o fato de que a lâmina de onda é um cristal birrefringente, i.e., possui diferentes refringências em direções ortogonais, conhecidos como eixo rápido e eixo lento [1-3]. Assim, quando o eixo rápido da LQO (nosso eixo de referência) está a $0^{\circ}$

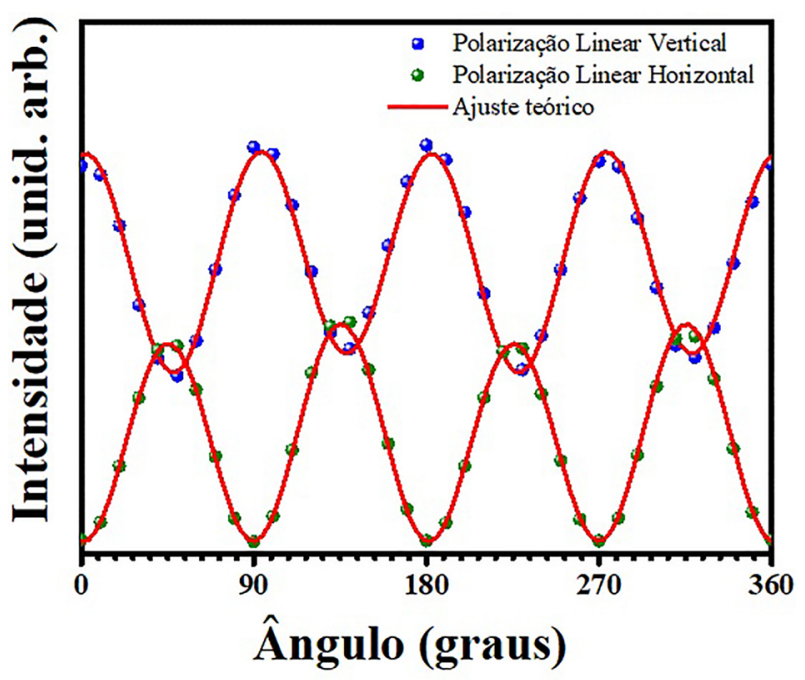

Figura 3: Curva de Stokes obtida para luz linearmente polarizada na direção vertical (esferas azuis) e horizontal (esferas verdes). Medida realizada utilizando-se no aparato experimental uma lâmina de quarto de onda comercial. As curvas vermelhas sólidas são os ajustes teóricos realizados com a equação (7).

$\left(90^{\circ}\right)$, em relação ao eixo vertical do referencial do laboratório, o campo elétrico da luz polarizada horizontalmente se propaga apenas no eixo lento (rápido) da LQO, o que não exerce nenhum tipo de efeito sobre sua polarização. Nesta condição a LQO se comporta como uma lâmina de vidro ordinária. Desta forma, quando atinge o analisador (um polarizador linear posicionado na direção vertical), a luz horizontalmente polarizada é completamente extinta e nenhum sinal é captado pelo detector. As posições dos máximos ocorrem quando a LPH tem componentes ortogonais com a mesma amplitude projetada sobre os dois eixos (rápido e lento) da LQO, i.e., quando o eixo rápido da LQO está em posições angulares múltiplas de $45^{\circ}$ em relação ao eixo de oscilação da LPH. Tais posições angulares são exatamente as posições intermediarias entre as duas posições de mínimo. Devemos notar que nesta configuração angular a LPH é transformada em luz circularmente polarizada, de modo que, quando incide sobre o analisador esta possui uma de suas componentes extinta e a outra transmitida, fornecendo assim a maior intensidade permitida para este estado de polarização, que corresponde à metade da intensidade total do feixe $[2,9]$. O resultado desta combinação de ações da lâmina de onda e do analisador sobre a luz polarizada fornece o padrão cossenoidal, com períodos de $90^{\circ}$ esperado da equação (7) quando $\mathrm{S}_{1} \approx \mathrm{S}_{0}>0$ e $\mathrm{S}_{2}=\mathrm{S}_{3} \sim 0$ Os resultados experimentais obtidos foram $\frac{S_{1}}{S_{0}} \cong 0,945( \pm 0,004)$, $\frac{S_{2}}{S_{0}} \cong-0,033( \pm 0,007)$ e $\frac{\mathrm{S}_{3}}{\mathrm{~S}_{0}} \cong-0,044( \pm 0,004)$. O grau de polarização estimado foi de $\mathrm{P}=0,947( \pm 0,004)$

A curva de Stokes para a LPV mostra um comportamento similar, mas com um mínimo de intensidade não nulo defasado de $45^{\circ}$ em relação a curva de Stokes obtida para a LPH (esferas azuis na Figura 3a). Assim, quando as posições angulares da LQO são múltiplos de 
$90^{\circ}$, o campo elétrico da LPV também oscila apenas sobre o eixo com uma única refringência (rápido ou lento) da LQO. Todavia, o estado de polarização não alterado agora é transmitido pelo analisador que, como dito antes, é ajustado na direção vertical. Por outro lado, quando a LPV se torna circularmente polarizada, o que ocorre quando o eixo rápido da LQO está a $45^{\circ}$ com a direção vertical, apenas metade da intensidade total é transmitida uma vez que uma das componentes será extinta pelo analisador. Tal fato gera um mínimo não nulo, equivalente ao máximo obtido para a curva de Stokes obtida para a LPH. Novamente obtém-se da combinação de ações da lâmina de onda e do analisador sobre a luz polarizada o padrão cossenoidal, com períodos de $90^{\circ}$ esperado da equação (7) quando $\mathrm{S}_{1} \approx \mathrm{S}_{0}<0$ e $\mathrm{S}_{2}=\mathrm{S}_{3}=0$ Os valores de $\frac{\mathrm{S}_{1}}{\mathrm{~S}_{0}} \cong-1,06( \pm 0,02), \frac{\mathrm{S}_{2}}{\mathrm{~S}_{0}} \cong-0,02( \pm 0,02)$ e $\frac{\mathrm{S}_{3}}{\mathrm{~S}_{0}} \cong-$ $0,05( \pm 0,01)$ foram experimentalmente obtidos para esta configuração, enquanto o grau de polarização estimado foi de $\mathrm{P}=1,09( \pm 0,02)$.

As curvas de Stokes medidas para a luz polarizada linearmente a $\pm 45^{\circ}$ são apresentadas na Figura 4 e verifica-se que estas possuem comportamento senoidal com período de $90^{\circ}$.

É também observado que estas, assim como as curvas obtidas para LPH e LPV, também possuem fases invertidas, com o máximo de uma correspondendo ao mínimo da outra e vice-versa, o que pode ser entendido como um deslocamento de fase também de $45^{\circ}$. Todavia, diferentemente da LPH e LPV, as curvas obtidas para polarização linear $\pm 45^{\circ}$ sempre apresentam uma intensidade de mínimo diferente de zero. A intensidade mínima, diferente de zero, é esperada, uma vez que para polarizações a $\pm 45^{\circ}$ sempre há uma componente de polarização que é transmitida pelo analisador (posicionado na direção vertical do referencial do laboratório). Isto ocorre,

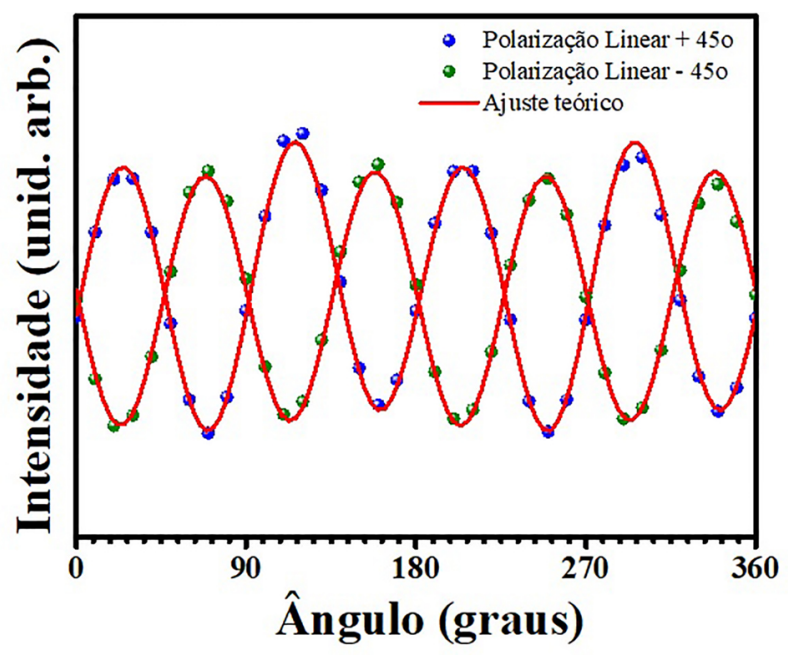

Figura 4: Curva de Stokes obtida para luz linearmente polarizada na direção $+45^{\circ}$ (esferas azuis) e $-45^{\circ}$ (esferas verdes). Medida realizada utilizando-se no aparato experimental uma lâmina de quarto de onda comercial. As curvas vermelhas são ajustes teóricos realizado com a equação (7). pois, para luz linearmente polarizada a $\pm 45^{\circ}$ a LQO, quando rotacionada, passa por posições angulares que geram desde luz circularmente polarizada (em múltiplos de $90^{\circ}$ iniciando pelo $0^{\circ}$ ) a luz linearmente polarizada a $\pm 45^{\circ}$, que ocorre quando a posição angular da lâmina é $45^{\circ}$. A única diferença entre a luz polarizada circularmente e a polarizada linearmente a $\pm 45^{\circ}$ é a diferença de fase entre as componentes ortogonais, o que não é detectado pelo analisador. Tal fato justifica a ausência de deslocamento na escala de intensidade observado para a LPH e LPV e explica por que as intensidades medidas para posições angulares múltiplas de $45^{\circ}$ (iniciando-se do $0^{\circ}$ ) são aproximadamente as mesmas. Os máximos (mínimos) são obtidos quando a LQO gera uma luz de saída elipticamente polarizada, com semieixo maior na direção de transmissão (extinção) do analisador. O comportamento experimental observado está em perfeito acordo com a equação (7), sendo este padrão esperado quando $\mathrm{S}_{2} \approx \mathrm{S}_{0}$ e $\mathrm{S}_{1}=\mathrm{S}_{3} \approx 0$, sendo $\mathrm{S}_{2}>0$, para luz polarizada $\mathrm{a}+45^{\circ}$ e $\mathrm{S}_{2}<0$ para $-45^{\circ}$. Os valores obtidos experimentalmente foram $\frac{\mathrm{S}_{1}}{\mathrm{~S}_{0}} \cong 0,2( \pm 0,01), \frac{\mathrm{S}_{2}}{\mathrm{~S}_{0}} \cong 0,94( \pm 0,1)$ e $\frac{\mathrm{S}_{3}}{\mathrm{~S}_{0}} \cong 0,06( \pm 0,01)$ para luz linearmente polarizada a $+45^{\circ}$ e $\frac{\mathrm{S}_{1}}{\mathrm{~S}_{0}} \cong 0,07( \pm 0,01), \frac{\mathrm{S}_{2}}{\mathrm{~S}_{0}} \cong-1,10( \pm 0,01)$ e $\frac{\mathrm{S}_{3}}{\mathrm{~S}_{0}} \cong 0,01$ $( \pm 0,01)$ para luz linearmente polarizada a $-45^{\circ}$. Os graus de polarização estimados foram $\mathrm{P}=0,96( \pm 0,01)$ para luz à $+45^{\circ}$ e $\mathrm{P}=1,10( \pm 0,02)$ para luz à $-45^{\circ}$.

Por fim, é importante notar que variações periódicas nas amplitudes de máximo e mínimo são observadas nas Figuras (3) e (4). De acordo com a equação (7), tais variações não existiriam se o feixe investigado fosse totalmente polarizado com um único estado de polarização. Todavia, todos os nossos resultados mostram que os feixes analisados possuem componentes, com pequena amplitude, nos outros estados de polarização possíveis. Essas pequenas amplitudes modulam a série de Fourier truncada (equação (7) ) que descreve o experimento em mais de uma frequência o que leva ao padrão observado.

\subsection{Superposição linear dos parâmetros de Stokes}

Com o objetivo de entender a superposição linear explícita na equação de Stokes (equação (7)), luz com polarização elíptica foi investigada. Isto foi obtido através do uso de um polarizador comercial de câmera fotográfica dito circular que gerou um estado de polarização elíptico que resultou na curva de Stokes mostrada na Figura 5 (esferas cinzas). Os parâmetros relativos de Stokes obtidos a partir do ajuste dos dados experimentais mostrados na Figura 5 (curva sólida preta) indicam que a luz é composta por luz circularmente polarizada a direita $\left(\frac{\mathrm{S}_{3}}{\mathrm{~S}_{0}}=0,916\right) \mathrm{e}$ luz linearmente polarizada na vertical $\left(\frac{\mathrm{S}_{1}}{\mathrm{~S}_{0}}=-0,312\right)$, e na direção $+45^{\circ}\left(\frac{\mathrm{S}_{2}}{\mathrm{~S}_{0}}=0,172\right)$. Os parâmetros de Stokes obtidos mostram que a luz apresenta uma polarização elíptica, com a elipse ligeiramente inclinada em relação ao eixo vertical. 


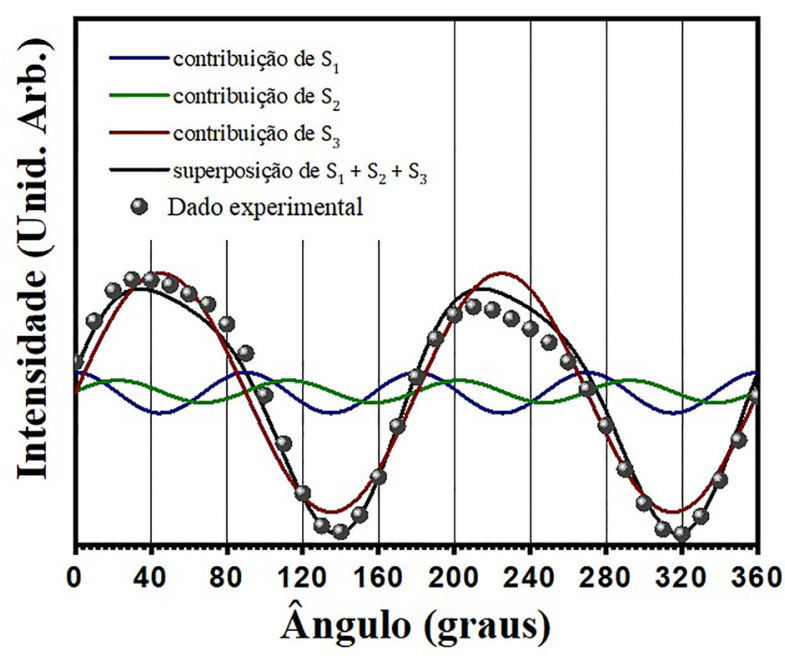

Figura 5: Curva de Stokes obtida para luz com polarização elíptica produzida com um polarizador circular comercial de câmera fotográfica (esferas cinzas). Curva de Stokes obtida para luz linearmente polarizada na vertical $\left(\frac{\mathrm{S}_{1}}{2}(1+\cos 4 \theta)\right.$ linha sólida azul marinho) linearmente polarizada a $+45^{\circ}\left(\frac{\mathrm{S}_{2}}{2} \sin 4 \theta\right.$, linha sólida verde oliva) e circularmente polarizada à direita $\left(\mathrm{S}_{3} \sin 2 \theta\right.$, linha sólida vinho). Curva resultante da superposição das três componentes de polarização (equação 7 , linha sólida preta).

Percebe-se que fenomenologicamente tal processo não é fácil de ser explicado a partir da ideia de elipse de polarização. Todavia, o uso do formalismo de Stokes e a ideia de superposição simplificam substancialmente a tarefa. Tal fato é ilustrado na Figura 5 onde os gráficos referentes às componentes linear vertical (curva sólida azul marinho), linear $+45^{\circ}$ (curva sólida verde oliva), e circular a direita (curva sólida vinho) são apresentados juntamente com o resultado de sua superposição. As curvas na Figura 5 para polarizações puramente linear e circular foram obtidas da equação (7), supondo as outras contribuições nulas. Os valores dos parâmetros de Stokes utilizados para construir teoricamente as curvas referentes as contribuições das polarizações fundamentais na Figura 5 foram obtidos do ajuste dos dados experimentais. Nota-se claramente que a curva de Stokes é resultante da superposição das três componentes de polarização (linha sólida preta na Figura 5). De fato, podemos pensar nos parâmetros de Stokes como uma base de estados de polarização, obtidas a partir de um observável físico mensurável (a intensidade da luz), com o qual podemos descrever qualquer estado de polarização da luz, inclusive o estado não polarizado que é obtido quando $\mathrm{S}_{0} \neq 0$ e $\mathrm{S}_{1}=\mathrm{S}_{2}=\mathrm{S}_{3}=0$

\subsection{Experimento de baixo custo para verificação do formalismo de Stokes}

Embora seja possível haver lâminas de onda comerciais em laboratórios avançados de ensino de física, sabemos que seu custo elevado inviabiliza seu uso rotineiro nesses laboratórios. Inspirados por essa realidade e pela referência [22], testamos o procedimento experimental aqui apresentado com uma lâmina de quarto de onda caseira fabricada com fita adesiva. Isto é possível uma vez que as fitas adesivas possuem birrefringência proveniente tanto de seu processo de fabricação quanto do seu manuseio, associada a anisotropia mecanicamente induzida por estiramento, ao ser retirada do rolo. O parâmetro a ser controlado é o número de camadas de fita [22]. Estas são adicionadas até obter-se um dispositivo que, para o comprimento de onda utilizado $(670 \mathrm{~nm})$, funcione como uma lâmina de quarto de onda [22]. Para testarmos o comportamento birrefringente da fita adesiva, utilizamos um aparato que consiste em transmitir o feixe de luz por dois polarizadores em série. O primeiro, fixo na vertical, funciona como polarizador da luz, enquanto o segundo, logo antes do detector, é rotacionado em torno do eixo de propagação do feixe de luz, e funciona como o analisador de intensidades lineares em vários planos de polarização. Quando nenhum objeto polarizante é colocado entre os dois polarizadores, o experimento fornece uma curva de intensidade versus ângulo entre os dois eixos de polarização, conhecida como Lei de Malus [5, 9], veja quadrados pretos no Figura 6. Esta lei afirma que a intensidade do feixe transmitido através de dois polarizadores segue uma lei cossenoidal quadrática com o ângulo relativo entre os dois polarizadores. Todavia, se uma lâmina de quarto de onda, para o comprimento de onda transmitido, é posicionada com eixo rápido a $45^{\circ}$ do eixo do analisador entre os dois polarizadores, a intensidade de luz transmitida pelo analisador é constante para todos os ângulos de rotação. Isto ocorre, pois o primeiro par de elementos (polarizador e lâmina) gera luz circularmente polarizada, cuja isotropia espacial de intensidades permite o analisador filtrar a mesma intensidade em qualquer direção [22]. Ao realizar este teste, observamos que três camadas de

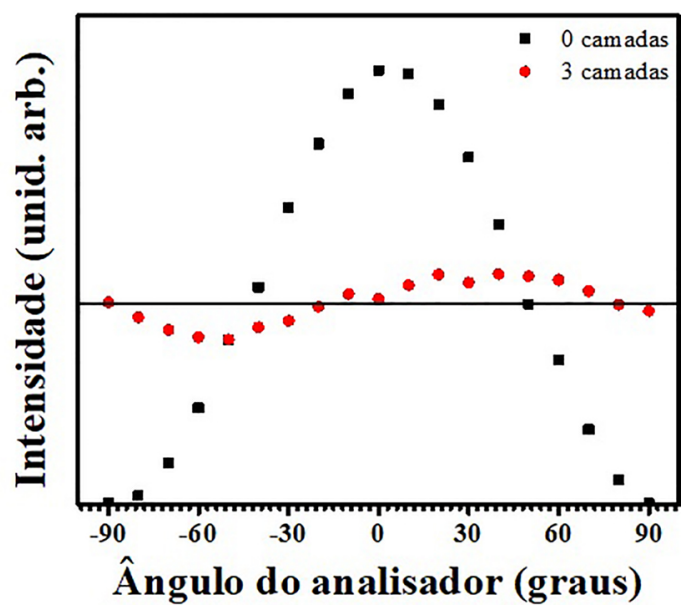

Figura 6: Intensidade versus ângulo entre os eixos de polarização para: (i) feixe transmitido somente por dois polarizadores em série (quadrados pretos); (ii) feixe transmitido pelo mesmo par de polarizadores, mas com uma lâmina birrefringente, de três camadas de fita adesiva com o eixo rápido à $\sim 45^{\circ}$ da vertical, inserida entre os polarizadores (círculos vermelhos). $O$ ângulo zero foi tomado onde os dois eixos de polarização são alinhados (máxima transmissão) 
fita adesiva produzem, aproximadamente, uma lâmina de quarto de onda em $670 \mathrm{~nm}$, veja círculos vermelhos na Figura 6. O eixo rápido das camadas de fita foi estimado como sendo perpendicular à direção de estiramento da fita, devido ao processo de estiramento [5].

Uma vez obtida nossa lâmina de quarto de onda caseira utilizamos a mesma para obter curvas de Stokes, como feito posteriormente. Na Figura 7, apresentamos curvas de Stokes obtidas para Luz linearmente polarizada na vertical e a $-45^{\circ}$.

Nota-se uma boa concordância qualitativa entre as curvas medidas com a lâmina de fita adesiva (Figura 7) e as curvas de Stokes medidas com a lâmina comercial (Figuras 3 e 4 ). Os valores encontrados de $\frac{\mathrm{S}_{1}}{\mathrm{~S}_{0}} \approx-0,86$ $( \pm 0,05), \frac{\mathrm{S}_{2}}{\mathrm{~S}_{0}} \approx-0,13( \pm 0,04)$ e $\frac{\mathrm{S}_{3}}{\mathrm{~S}_{0}} \approx 0,04( \pm 0,02)$ para luz linearmente polarizada na vertical. Para a luz linearmente polarizada a - $45^{\circ}$ os valores encontrados são: $\frac{\mathrm{S}_{1}}{\mathrm{~S}_{0}} \approx-0,39$ $( \pm 0,03) \frac{\mathrm{S}_{2}}{\mathrm{~S}_{0}} \approx-1,49( \pm 0,05)$ e $\frac{\mathrm{S}_{3}}{\mathrm{~S}_{0}} \approx-0,02( \pm 0,02)$. Os graus de polarização obtidos são: $\mathrm{P} \approx 0,87$ para luz linearmente polarizada na vertical e $\mathrm{P} \approx 1.54( \pm 0,04)$ para luz linearmente polarizada $-45^{\circ}( \pm 0.04)$. Tais valores possuem um erro intrínseco significativo, comparados ao valor unitário que os parâmetros relativos e o grau de polarização deveriam ter. Estes erros são atribuídos à natureza não-ideal da lâmina de fita adesiva como lâmina de quarto de onda ideal. Além disso, a equação (7) é construída assumindo que a retardância da lâmina seja de $90^{\circ}$ (ou 0.25 ondas) e que não há a incerteza na verdadeira posição $\theta$ do eixo rápido da lâmina Tais fatores são responsáveis por transferir as incertezas das características da lâmina para dentro dos valores dos Parâmetros de Stokes. Dessa maneira, o erro quantitativo obtido para lâmina de fita adesiva pode ser reduzido com a construção de lâminas com o comportamento mais próximo de uma lâmina de quarto de onda, como os apresentados na referência [22]

\section{Conclusões}

Neste trabalho uma proposta de prática experimental para ser utilizada em laboratórios avançados de ensino de óptica é apresentada, sendo sua condução um estudo de caso baseado em metodologia de aprendizado por projeto.
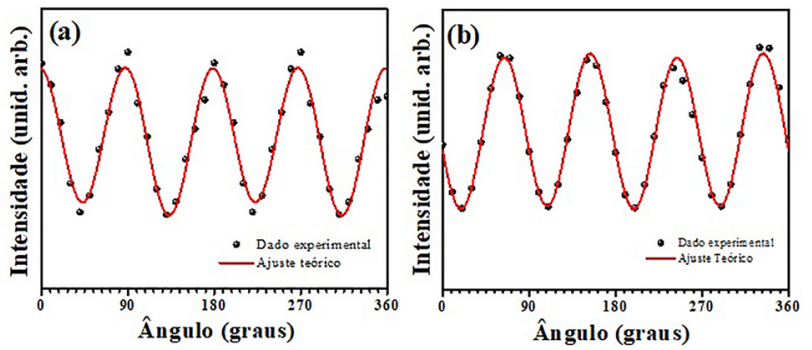

Figura 7: Curva de Stokes obtidas para luz linearmente polarizada (a) na vertical e (b) à $-45^{\circ}$. Os experimentos foram realizados com a lâmina de fabricação caseira feita com fita adesiva. As esferas pretas são os pontos experimentais e as linhas vermelhas são os ajustes obtidos com a equação (7).
Neste, os estudantes foram capazes de desenvolver toda a fundamentação teórica do problema que serviu de base para a construção da prática capaz de determinar os parâmetros de Stokes para estados de polarização distintos. As curvas de Stokes relacionadas a cada estado de polarização foram investigadas e sua fenomenologia discutida. Todos os resultados experimentais estão de acordo com a teoria apresentada, dada a discussão sobre os erros associados, implicando no bom funcionamento da prática experimental. A partir da geração e da investigação de luz elipticamente polarizada, fomos capazes de demonstrar que o formalismo de Stokes obedece ao princípio de superposição sendo a polarização elíptica (a mais geral possível) sendo formada a partir da superposição linear das polarizações base (linear vertical e horizontal; linear $\pm 45^{\circ}$, e circular a direita e a esquerda). Desta forma temos os parâmetros de Stokes como uma base de estados de polarização, obtidas a partir de um observável físico mensurável (a intensidade da luz), com o qual podemos descrever qualquer estado de polarização da luz. Destacamos também as medidas realizadas com lâminas de onda caseiras produzidas por anisotropia mecanicamente induzida em fitas adesivas. Também vale ressaltar que a importância do formalismo de Stokes e da técnica da lâmina de quarto de onda rotatória reside em suas vantagens de cunho prático-experimental e de completeza-descritiva. Com este sistema, por exemplo, não existem problemas de medidas com sistemas sensíveis à polarização de luz incidente, como os que possuem grades de difração, uma vez que o último elemento polarizador é mantido fixo, o que elimina a necessidade de calibrações. Além disso, esta técnica é capaz de diferenciar as porções lineares e circulares da luz, descrevendo completamente a elipse de polarização. É importante frisar que a Lei de Malus não consegue discernir uma luz circularmente polarizada de uma não polarizada. Por fim, com o formalismo de Stokes, o estado, logo a elipse de polarização, é completamente e univocamente descrito, o que permite seu emprego para investigação em ciências dos materiais e no estudo espectroscópico de fenômenos anisotrópicos.

\section{Agradecimentos}

Os autores são gratos ao CNPq (Grant No. 309050/20170), FAPESPA e CAPES (AUXPE 88881.159129/2017-01) bem como a Pró-reitoria de extensão da Universidade Federal do Pará pelo apoio dado a esta pesquisa. Os autores também estão em dívida com o Prof. Sanclayton Geraldo Carneiro Moreira do Programa de Pós-graduação em Física da Universidade Federal do Pará pelo uso de equipamentos ópticos. PTA agradece ao National Science Foundation (NSF)

\section{Referências}

[1] M. Young, Óptica e Lasers (EDUSP, São Paulo, 1998). 
[2] G.R. Fowles, Introduction to Modern Optics (Dover Publications Inc., New York, 1975).

[3] I.R. Kenyon, The light fantastic: A modern introduction to classical and quantum optics (Oxford University Press, Oxford, 2008).

[4] A.C. Melissinos, Experiments in Modern Physics (Acamedic Press, Inc., San Diego, 1966).

[5] E. Hecht, Optics (Addison Wesley, San Francisco, 2002), $4^{\mathrm{a}}$ ed.

[6] M. Herzberger, Modern geometrical optics (Interscience Publishers, Inc., New York, 1958).

[7] N.V. Tkachencko, Optical spectroscopy: Methods and instrumentations (Elsevier, Amsterdam, 2006).

[8] W. Demtröder, Laser spectroscopy: Basic concepts and instrumentation (Springer, New York, 2003), $3^{\mathrm{a}}$ ed.

[9] H.M. Nussenzveig, Curso de Física Básica: Ótica, Relatividade, Física Quântica (Editora Blucher, São Paulo, 1998), v. 4.

[10] D. Halliday e R. Resnick, Física (LTC editora, Rio de Janeiro, 1984), v. 4, $4^{\mathrm{a}}$ ed.

[11] R.C. Viscovini, Rev. Bras. Ens. Fis. 22, 143 (2000).

[12] J.L.P. Ribeiro e M.F.S. Verdeaux, Rev. Bras. Ens. Fis. 34, 4403 (2012).

[13] F.R. Henrique, N.B. Tomazio, R.G.T. Rosa, A.M. de Souza, C.P. D'Almeida, L.F. Sciuti, M.R. Garcia e L. De Boni, Rev. Bras. Ens. Fis. 41, e20180223 (2019).

[14] T.I. Pasqualetto, E.A. Veit e I.S. Araujo, Rev. Bras. Educ. Ciên. 17, 551 (2017).

[15] M.P. Linhares e E.M. Reis, Ciênc. Educ. 14, 555 (2008).

[16] J.R.T. Reis, A.G. Rodrigues e N.M. Barbosa Neto, Rev. Bras. Ens. Fis. 41, e20180081 (2019).

[17] P. Alliprandini-Filho, R. A.Silva, N. M. Barbosa Neto e A. Marletta, Chem. Phys. Lett. 469, 94 (2009).

[18] P. Alliprandini-Filho, G.F. Borges, W.B. Calixto, I.H. Bechtold, A.A. Vieira, R. Cristiano, H. Gallardo, R.A. Silva, N.M. Barbosa Neto e A. Marletta, Chem. Phys. Lett. 487, 94 (2010).

[19] G.G. Dalkiranis, G. Gustavo, E.M. Therézio, G. Conte, H. Gallardo, I.H. Bechtold e A. Marletta, Phys. Rev. E. 98, 022702 (2018).

[20] F.C. Basílio, P.T. Campana, E.M. Therézio, N.M. Barbosa Neto, F.S. Spirau, R.A. Silva e O.N. Oliveira Jr., J. Phys. Chem. C. 120, 136 (2016).

[21] D. Goldstein, Polarized light (Marcel Dekker Inc., New York, 2003), $2^{\mathrm{a}}$ ed.

[22] N. Carlin, E.M. Szanto, W.A. Seale, F.O. Jorge, F.A. Souza, I.H. Bechtold e L.R. Gasques, Rev. Bras. Ens. Fis. 27, 349 (2005).

[23] D.J. Griffiths, Introduction to eletrodynamics (Prentice Hall Inc., New Jersey, 1999), $3^{\text {a }}$ ed.

[24] H.M. Nussenzveig, Curso de Física Básica: Eletromagnetismo, (Editora Edgard Blücher, São Paulo, 1997), v. 3.

[25] B. Schaefer, E. Collett, R. Smyth, D. Barrett e B. Fraher, Am. J. Phys. 75, 163 (2007).

[26] G.G. Stokes, Transac. Cambr. Philos. Soc. 9, 399 (1852).

[27] H.G. Berry, G. Gabrielse e A.E. Livingston, Appl. Opt. 16, 3200 (1977).

[28] H.G. Gutierre, M.S. Ribeiro, L.A.A. Pereira, G.K. Cruz, R.F. Turchiello e S.L. Gómez, Ver. Bras. Ens. Fis. 39, e3501 (2017).
[29] P.R. Bevington e D.K. Robison, Data reduction and error analysis (McGral Hill, New Tork, 2003). 